\title{
Cours de vision industrielle Contrôle qualité par caméra linéaire
}

\author{
Abdelmalik TALEB-AhMED ${ }^{*}$, Luc DuvieubourG ${ }^{* *}$ \\ * Laboratoire d'Analyse des Systèmes du Littoral \\ Université du Littoral Côte d'Opale \\ Bâtiment A - Rue F. Buisson \\ 62228 Calais cedex, FRANCE \\ Tél. : (33) 321465654 - Fax : (33) 321460680 , \\ ** Laboratoire d'Automatique I ${ }^{3} \mathrm{D}$ - CNRS FRE 2497 \\ Université des Sciences et Technologies de Lille \\ Cité Scientifique, Bâtiment P2 \\ 59655 Villeneuve d'Ascq cedex, FRANCE \\ Tél. : (33) 320434169 - Fax : (33) 320436567 \\ talebelasl-gw.univ-littoral.fr
}

\section{DESCRIPTION}

Ce cours de 2ème cycle s'inscrit dans le prolongement du cours de vision numérique. Le volume d'heures consacré au cours de vision industrielle varie d'une maîtrise à l'autre. Il est compris entre 15 et 24 heures. Nous présentons le cours que nous donnons dans le cadre de la maîtrise EEA. Il n'est pas question dans ce cours de reprendre les méthodes d'analyse ou de traitements de l'image. Cependant lorsqu'une application donnée en exemple nécessite un traitement particulier, non abordé dans le cours de traitements d'image, celui-ci sera développé.

Pour suivre ce cours il est indispensable d'avoir comme pré-requis le cours de traitement d'images. Dans une moindre mesure des notions d'informatique industrielle et de programmation sont utiles. De plus des notions d'optique de base seront utilisées dans ce cours de vision industrielle. On peut ainsi se rendre compte du spectre très large des connaissances à acquérir avant d'entreprendre la réalisation d'un système d'inspection visuelle.

\section{OBJECTIFS}

A la fin de ce cours, l'étudiant devrait être à l'aise avec les concepts de vision automatique en industrie et être en mesure de participer activement à des études de faisabilité d'implantation de systèmes de vision. Il devrait aussi être familier avec les recherches que sous-tendent ces démarches (bibliographie, rédaction technique, contact avec le milieu...).

\section{CONTENU}

L'accent est ici mis sur l'application de la vision au domaine, très vaste, de l'inspection industrielle le long de la ligne de production. On y présente une justification de l'automatisation et du contrôle des procédés comme outils indispensables à la poursuite de la qualité. Des considérations relatives aux types 
de matériaux, aux modes d'acquisition optique, aux méthodes de traitement du signal, aux schémas d'implantation pratique de systèmes d'inspection et aux caractéristiques de l'environnement industriel sont également présentées. Lorsque cela est nécessaire le cours est illustré par des applications industrielles, réalisées par les auteurs.

Cet enseignement fait appel aux travaux pratiques pour asseoir la présentation théorique sur une expérimentation concrète. Il s'appuie également sur une large base d'applications dans des domaines variés des sciences de l'ingénieur qui sont présentés en illustration.

\section{BIBLIOGRAPHIE}

Un tel cours est basé sur une revue extensive de la littérature courante. Des notes condensant ces articles seront mises à la disposition des étudiants.

\section{PREAMBULE}

Beaucoup d'entreprises industrielles utilisent aujourd'hui la vision pour automatiser des opérations fastidieuses de tri ou de contrôle à des cadences très rapides. Ainsi pour tenter de se rapprocher des contraintes industrielles, nous nous sommes basés pour la progression de ce cours sur la démarche suivie lors de l'installation d'un système de contrôle qualité par vision artificielle. Pratiquement, ce cours est assuré sous forme de petits séminaires. Nous abordons essentiellement les systèmes de contrôle utilisant les caméras linéaires. En parallèle avec cette progression nous donnons en exemple une étude réalisée par nos soins. Nous avons fait le choix de diviser cette progression en cinq étapes :

(1) Pertinence d'un contrôle par vision artificielle,

(2) Sélection des moyens matériels,

(3) Développement logiciel,

(4) Installation du système et validation du contrôle,

(5) Acceptation et pérennité du système.

Dans le point (1) nous nous posons la question de la nécessité d'un système de contrôle qualité par vision artificielle. Pour répondre à cette question nous discutons des possibilités et des limites des systèmes de vision artificielle appliqués au contrôle qualité. Les points (2) et (3) représentent l'essentiel de ce cours. Nous calculons alors sur des exemples concrets les éléments matériels qu'il est souhaitable d'utiliser. Nous discutons ensuite des algorithmes permettant d'effectuer le contrôle des produits en temps réel. Bien que dans certains cas, des calculs élémentaires suffisent à résoudre les problèmes posés, tout l'arsenal des méthodes du traitement d'image vient bien à point pour parvenir à extraire l'information pertinente. Le point (4) permet de s'apercevoir des problèmes et dans ce cas de modifier tout ou une partie du matériel ou encore un traitement logiciel. Par expérience, le dernier point reste le plus délicat puisqu'il fait intervenir des facteurs humains : au niveau du personnel de production chargé du fonctionnement au quotidien ainsi qu'au niveau du personnel du service "contrôle qualité" chargé du bon fonctionnement dans le temps du système.

Afin de rester concis nous présentons uniquement dans cet article les grandes lignes du cours, qui reprend les points ennoncés précédemment. En fin d'article nous donnons les textes des travaux pratiques que les étudiants effectuent à la suite du cours.

\section{Pertinence d'un contrôle par vision artificielle}

L'intérêt d'un système de vision industrielle est d'améliorer la productivité et la qualité de la production. Le contrôle par vision évite le travail fastidieux d'un contrôle manuel, et permet d'être plus rapide pour des tâches répétitives. Il s'agit de développer un système de vision rapide, fiable et ayant pour objectif le "zéro défaut". 
Á partir d'un cahier des charges, une étude de faisabilité va permettre de savoir si le système de vision souhaité est réalisable. Cette étude doit mettre en évidence les différents choix effectués pour réaliser le système, ainsi que les possibilités et les limites du système. Un système peut contrôler le produit fini, mais aussi les différentes étapes de fabrication. Il faut choisir l'endroit le plus judicieux pour placer son système de vision en tenant compte de l'environnement. L'étape majeure du système est l'acquisition de l'image. En effet, il sera plus difficile d'extraire une information d'une image de mauvaise qualité. Voilà pourquoi le choix de la caméra, de l'objectif et de l'éclairage est primordial. Le logiciel de traitement et d'analyse d'images doit être simple et convivial pour l'opérateur. Les algorithmes de traitements doivent être rapides et robustes. L'étude de faisabilité devra mettre en évidence les caractéristiques du système: disposition du système, distance entre la caméra et la scène, le champ couvert par un pixel, la taille du champ d'observation, tolérances.

En général on considère que la vision industrielle se prête bien au contrôle qualité lorsque :

- Les tâches sont répétitives; telles que la recherche de défauts sur de grandes surfaces de matériaux défilants.

- Les tâches sont simples et bien définies, et l'on connaît à l'avance les caractéristiques des scènes à observer et l'information à en extraire.

Inversement le contrôle qualité ne peut être envisagé par vision lorsque :

- Il est difficile pour un opérateur humain de décrire de façon simple les défauts ou que ceux-ci demandent un degré d'intelligence et de compréhension important.

- Les cadences de production dépassent les capacités de traitement des systèmes de vision. Ces capacitès ne sont compréhensibles par l'étudiant que s'il connaît l'offre du marché dans le domaine de la vision. Actuellement il existe fort peu de cas ou ces contraintes rendent impossible le contrôle par vision. Par contre, dans ces cas extrèmes les coûts peuvent devenir importants.

\subsection{Avantages du contrôle qualité par vision}

Les avantages des systèmes de vision par rapport à d'autres types de capteurs sont nombreux :

- Ils sont souples d'emploi et permettent une très grande variété d'utilisation. Par simple reprogrammation, un capteur de vision peut changer radicalement de type d'inspection et peut s'adapter instantanément à d'autres pièces.

- Ils permettent de réaliser simultanément plusieurs mesures, éventuellement de nature différente.

- Ils permettent des mesures sans contact et non destructives, avec une vitesse et une précision appréciables.

- Ils permettent de rendre objectives certaines mesures et de garantir ainsi la répétabilité de l'apparence des objets (constance de la couleur, de la forme, ...).

- Plus que tous dispositifs automatiques, ils permettent de remplacer des opérateurs humains dans des tâches fastidieuses qui requièrent attention et concentration.

\subsection{Définitions}

Afin de fixer les idées, nous rappelons également quelques définitions généralement utilisées dans la communauté de la vision industrielle.

La vision industrielle, appelé "Machine Vision" par les Anglo-Saxons, est une discipline appliquée de la vision artificielle qui emploie le traitement numérique d'images pour automatiser des tâches. 
Elle utilise des moyens électroniques et informatiques pour doter des machines et robots de capacités sensorielles analogues à la vision humaine. Autrement dit, elle utilise des caméras comme capteurs pour détecter des défauts sur des objets manufacturés, inspecter des pièces en cours de fabrication, les compter, les trier, les classer, les mesurer, ... à partir de leur apparence visuelle. Il faut néanmoins se garder d'imaginer une analogie trop profonde entre les performances de la vision humaine, capable d'explorer la structure spatiale de son environnement et d'y reconnaître des objets complexes et variés, connus ou inconnus, en deux dimensions comme en trois, et les capacités bien plus modestes des systèmes de vision industriels.

Inspecter un produit au sens de la vision industrielle consiste à déterminer s'il répond à un certain nombre de spécifications. Cela revient souvent à vérifier si un assemblage a été correctement réalisé, si les dimensions d'un produit sont respectées ou si son aspect extérieur est satisfaisant ou encore conforme par rapport à un modèle de référence.

On parle d'inspection implicite lorsque les défauts à détecter sont connus à l'avance car les produits examinés et leurs défaillances éventuelles sont parfaitement identifiés. Par exemple, la présence d'un bouchon sur une bouteille ou d'une étiquette sur un emballage, l'intégrité d'un afficheur à cristaux liquides, sont des éléments qui peuvent être identifiés par référence à un modèle.

L'inspection est explicite quand les défauts recherchés sont mal définis ou qu'ils sont même imprévisibles. L'aspect visuel d'un état de surface, d'une déchirure, d'une fissure ou d'une tache sont difficilement prévisibles. L'opérateur humain est très performant pour détecter ces types de défauts qui ne peuvent être décrits a priori par des paramètres explicites. Il s'agit donc d'une inspection explicite dont l'automatisation est souvent difficile et qui ouvre la porte d'un domaine de recherche actif.

\subsection{Applications typiques}

A titre d'exemple, nous présentons dans cette partie du cours des secteurs industriels concernés par le contrôle qualité par vision linéaire.

On peut citer notamment les procédés où défilent à grande vitesse des matériaux en feuille et en rouleaux qui doivent être exempts de défauts tels que taches, griffes, trous, plis, reliefs et autres hétérogénéités, cf. figure 10.

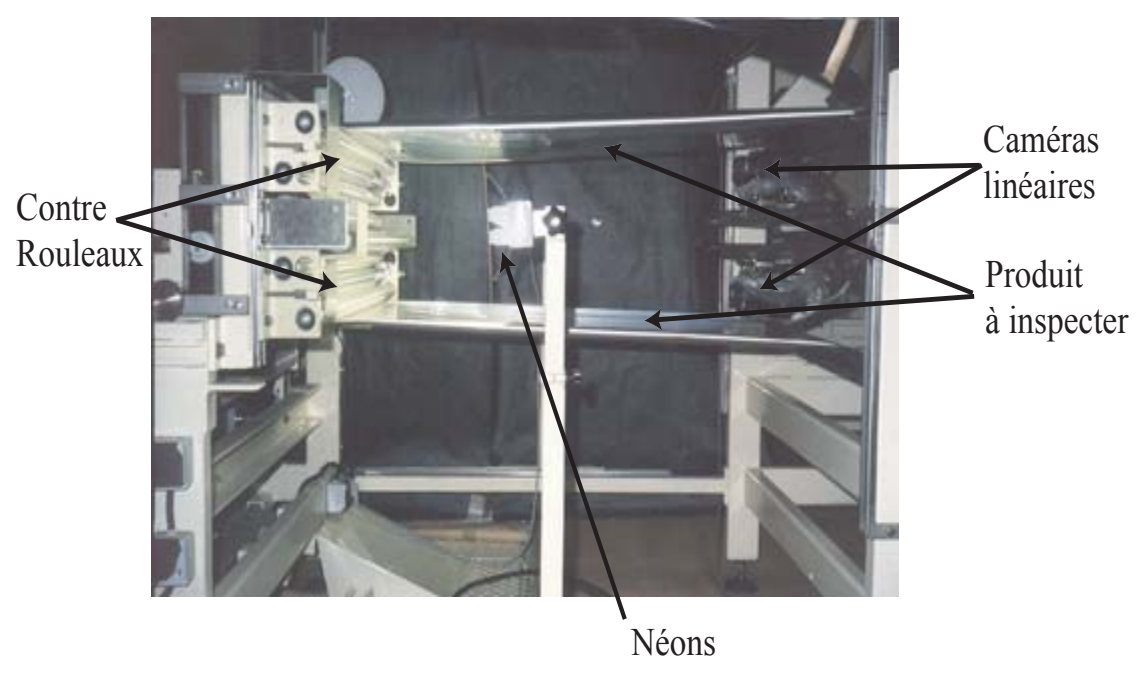

FIG. 1: Contrôle qualité sur produit en défilement

Ce type d'application se rencontre dans l'industrie papetière, dans la sidérurgie, la fabrication du verre 
plat, l'industrie textile, ... Dans les cas les plus simples, il s'agit de surfaces parfaitement lisses et uniformes où il suffit de détecter des changements de couleur ponctuels; dans d'autres, on est confronté à des textures périodiques ou non, et il est plus délicat de séparer les variations normales et accidentelles.

\section{Sélection des moyens matériels}

Dans cette partie du cours nous présentons les différents composants intervenant dans un système de contrôle qualité par vision linéaire. Nous donnons pour chacun de ces composants les éléments qui déterminent son choix par rapport aux contraintes du cahier des charges. Nous passons en revue également les techniques optiques de base permettant d'obtenir des images de bonne qualité. Nous commençons par le composant le plus important, c'est à dire, l'éclairage.

\subsection{Choix de l'éclairage}

L'éclairage est un élément déterminant dans un système de contrôle par vision industrielle. En fonction de la technique d'éclairage employée, on pourra faire ressortir certains défauts.

\subsubsection{Diascopique ou Rétro-éclairage}

Dans ce cas la caméra et l'éclairage sont de part et d'autre du produit à inspecter. Le rétro-éclairage permet de mettre en évidence le contour d'un objet, son ombre. On l'utilise pour faire des mesures ou détecter des imperfections (trous, déchirures, ...).

\subsection{2 Épiscopique}

Dans cette configuration la caméra et l'éclairage sont du même coté par rapport au produit à inspecter. En fonction du type de défaut que l'on veut faire ressortir, on trouve distingue alors différentes stratégies d'éclairage :

- Éclairage diffus : L'intérêt d'un éclairage diffus est de donner une image qui n'est pas perturbée par des ombres ou des reflets. Un tel éclairage s'utilise avec un objet contrasté et marqué. On peut utiliser une sphère intégratrice qui donne un éclairage homogène.

- Éclairage directionnel (Bright field): Un éclairage directionnel permet de mettre en valeur une caractéristique de l'objet.

- Éclairage rasant (Dark field) : Un éclairage rasant peut faire ressortir le relief (rayures, bosses...).

- Éclairage laser : Projeter une ligne laser va permettre de localiser des contours ou des points caractéristiques de l'objet.

- Polarisation : Polariser la lumière revient à créer une direction privilégiée. La polaristion atténue les reflets parasites de l'objet. Si on a un défaut en relief, la lumière peut perdre son axe de polarisation, alors le défaut apparaîtra sur le capteur.

\subsubsection{Les sources d'éclairage}

- Les lampes halogènes sont souvent utilisées comme sources avec des fibres optiques qui transportent la lumière jusqu'à un système d'éclairage déporté. Cette technologie est souple d'utilisation: l'intensité lumineuse est réglable et la forme peut être ponctuelle, linéaire, annulaire ou 
surfacique (rétro-éclairage). Par contre, la durée de vie de ces lampes est relativement faible, de l'ordre de 1000 heures.

- Les éclairages fluorescents offrent une grande uniformité, une durée de vie importante et un faible échauffement. Leur fonctionnement repose sur des décharges périodiques d'électrons à travers le gaz contenu dans l'ampoule ce qui provoque la fluorescence. Ces décharges sont initiées par un ballast et leur fréquence doit être élevée. Les lampes fluorescentes sont exploitées en éclairage linéaire et annulaire.

- Les éclairages à LED fournissent une lumière uniforme et ont une durée de vie de plusieurs années. Elles fournissent chacune une petite quantité de lumière et il faut donc en aligner plusieurs pour avoir un système d'éclairage. La lumière produite est uniforme et a en général une longueur d'onde précise, infrarouge, rouge, vert.... Des systèmes produisant une lumière blanche existent néanmoins. Compte tenu de leur temps de réponse très court, les LED peuvent être utilisées comme source stroboscopique. L'une des caractéristiques les plus remarquables des LED est leur durée de vie de plusieurs années. L'assemblage de LED permet d'obtenir des dispositifs linéaires, annulaires ou surfaciques.

La plupart des techniques d'éclairage décrites ci-dessus sont illustrées dans le cours par des exemples d'applications. Á travers ces exemples nous voulons montrer l'importance et la difficulté de la définition de l'éclairage dans un système de vision. De nombreux paramètres entrent en ligne de compte. Les plus importants sont liés à l'objet étudié : l'état de surface, les caractéristiques à accentuer, celles qui sont à gommer... Nous voyons que les stratégies d'éclairage sont nombreuses et reposent sur l'orientation des rayons lumineux, la technologie et la géométrie du système. Dans la cadre de la première séance de travaux pratiques l'étudiant devra mettre en oeuvre une de ces techniques en fonction du type de défauts à détecter.

Pour l'étudiant intéressé par les techniques d'éclairage ainsi que sur l'offre dans ce domaine, voici une adresse de sites web : www.cs.cf.ac.uk/User-bin/M.R.F.Lewis/lightidx.cgi

L'université de Cardiff propose un site très complet répertoriant l'ensemble des techniques d'éclairage avec leurs particularités, leurs applications ainsi que les caméras et optiques appropriées.

\subsubsection{Autres considérations}

L'image qui arrive au capteur de la caméra résulte de l'interaction de la lumière avec la scène observée. Pour minimiser les traitements logiciels, il est intéressant d'obtenir un éclairage distribué de façon parfaitement uniforme et homogène. Malheureusement, il est très difficile en pratique d'assurer cette uniformité, et la constance de l'intensité au cours du temps. De plus, des effets tels que les ombres portées, qui occultent une partie de la scène, ou les reflets, qui éblouissent le capteur, détruisent par endroit le signal. Ces défauts sont d'autant plus vrais que le champ de vue de la caméra est important. Il est plus facile d'assurer cette uniformité de l'éclairage pour une caméra linéaire, dont le champ de vue correspond à une ligne sur le produit. Les ombres peuvent être minimisées par la disposition judicieuse des sources, à moins que leur présence ne soit désirée. Dans le cas des système de vision linéaire la non-uniformité de l'éclairage peut être compensée facilement par la carte d'acquisition, par un calibrage de l'échelle des niveaux de gris pour chacun des pixels.

Il faut être vigilant sur la fréquence de fonctionnement du système d'éclairage, ce qui peut être préjudiciable pour la prise d'images. Par exemple, dans le cas d'un éclairage par tubes au néon il est impératif que la fréquence de l'alimentation des tubes soit supérieure à la fréquence d'acquisition des images-ligne, pour éviter le phénomène de battement. 


\subsection{Choix de l'optique}

Nous présentons dans ce paragraphe la démarche qui conduit au choix de l'objectif. Ce choix est, dans un premier temps, conditionné par la distance focale.

\subsubsection{Caractéristiques des objectifs}

- L'angle de vue.

- Le nombre d'ouverture: Le nombre d'ouverture $\mathrm{F}=\mathrm{f} / \mathrm{d}$ avec $\mathrm{f}$ la focale et $\mathrm{d}$ le diamètre de la pupille d'entrée. Nombres d'ouvertures normalisés : 1,4 - 2 - 2,8 - 4 - 5,6 .

Plus le nombre d'ouverture augmente, plus la quantité de lumière diminue au travers de l'objectif ( $d$ diminue), plus la profondeur de champ est grande. A l'inverse, plus le nombre d'ouverture diminue, plus la quantité de lumière augmente ( $d$ augmente), plus la profondeur de champ diminue, plus l'objectif est rapide. Si le nombre d'ouverture est petit, alors le diamètre de la pupille d'entrée est grand. On s'éloigne alors des conditions de Gauss (rayons proches de l'axe optique), ce qui peut entrainer des aberrations géométriques et chromatiques.

Remarque: on peut parfois voir la notation f/xx sur l'objectif. xx est le nombre d'ouverture. On voit aussi la notation 1:4 équivalent à f/4.

Pour résumer, le nombre d'ouverture influe sur :

- la quantité de lumière,

- la profondeur de champ,

- la résolution.

- La distance focale : Le choix de la focale de l'objectif est déterminé à partir de la taille du capteur, de la taille de l'objet et de la distance à laquelle est vu l'objet.

- La profondeur de champ d'un objectif représente la distance entre deux points telle que tout objet entre les deux points soit vu net par le capteur et au delà des deux points soit vu flou. La profondeur de champ augmente quand :

- Le nombre d'ouverture augmente.

- La distance objet/objectif augmente.

- La focale diminue.

- Le grandissement diminue.

- Á agrandissement égal, la profondeur de champ reste constante à focale différente.

\subsubsection{Autres considérations}

- Pouvoir séparateur et FTM : La fonction de transfert de modulation FTM (ou MTF = Modulation Transfer Function) est une courbe qui relie la variation de contraste de l'image à la fréquence spatiale de l'objet (définie par le nombre de paires de lignes par millimètres). L'objectif doit avoir un pouvoir séparateur supérieur à celui du capteur. On peut le mesurer avec une mire.

- Distorsion: Plus la focale diminue, plus le risque de distorsions sera important car on aura un grand angle.

- Un peu de vocabulaire

- Objectif grand angle : petite focale $(<50 \mathrm{~mm})$, grand champ.

- Téléobjectif : grande focale ( $>50 \mathrm{~mm})$, petit champ. 
- Télécentrique : grandissement constant quelle que soit la distance objet-objectif, donc pas de perspective.

- Doubleur de focale: c'est une lentille qui se place entre l'objectif et le capteur qui double la focale. Le grandissement est multiplié par deux mais ceci pose quelques problèmes: augmentation de la distorsion liée au grandissement, augmentation du nombre d'ouverture, diminution de la profondeur de champ.

- Focale variable : les zooms sont des objectifs à focale variable. Une focale variable permet de choisir le meilleur cadrage sans changer d'objectif. On joue sur le grossissement de l'objectif. Le manque de luminosité par rapport à une focale fixe peut être un inconvénient.

- Tirage optique (Back Focal Length) : distance entre le plan focal image et la lentille de sortie de l'objectif.

- Tirage mécanique (Flange Back) : distance entre la face mécanique d'appui de la monture de l'objectif et la plan du capteur.

Les imperfections et mauvais réglages de l'optique introduisent des distorsions dues aux aberrations et à l'effet de perspective, du flou dû à la défocalisation ou à la diffraction, de la non-uniformité de luminosité transmise. Ces distorsions peuvent être minimisées par des connaissances d'optique de bases. Cependant il faudra faire un compromis entre la distance caméra produit, la puissance de l'éclairage, la période d'acquistion et l'ouverture de l'objectif. Par ailleurs il est toujours possible de corriger ces distorsions de façon logicielle par calibration géométrique du champ visuel. En observant l'image d'une mire de forme connue, on peut mesurer la distorsion et la compenser au moyen de la transformation inverse. Dans certains cas, c'est l'objet mesuré lui-même qui peut servir de mire.

\subsection{Choix de la caméra}

Suivant le produit à inspecter, nous choisissons la caméra :

- Linéaire ou matricielle?

- Analogique ou numérique?

- Monochrome ou couleur(Mono ou tri-CCD)?

Les caméras linéaires: Ces caméras fonctionnent sur le même principe qu'un scanner ou un fax. Une caméra linéaire est constituée d'une seule rangée de capteurs. C'est le mouvement relatif de la scène par rapport à la caméra qui va reconstituer une image par balayage. Ces capteurs permettent d'atteindre des vitesses et des résolutions élevées (plus de 8000 pixels sur une seule ligne). Les caméras linéaires sont idéales pour visualiser des objets en mouvement ou de grande dimension, ainsi que pour superviser des process continus (textile). Une application typique de ces caméras est la visualisation d'objets cylindriques: par rotation de l'objet autour de son axe de révolution, on obtient une visualisation plane de sa surface. Afin de reconstituer une image bien proportionnée, la caméra doit être synchronisée avec la mécanique d'avancement. Pour cela, on utilise un encodeur (fixé sur l'arbre moteur par exemple) afin de générer des signaux qui déclencheront l'acquisition. Durant une rotation, l'encodeur génère un nombre précis d'impulsions desquelles on peut déduire la distance parcourue.

Le choix entre une caméra analogique et une caméra numérique repose sur un compromis entre le coût et les performances. Les caméras numériques permettent d'atteindre des vitesses d'acquisition élevées, des images très précises et un excellent rapport signal/bruit. Les caméras analogiques sont basées sur une technologie éprouvée et sont donc plus répandues et moins chères. La plupart des modèles répondent à un standard ce qui simplifie leur paramétrage. La plupart des caméras numériques ont leur résolution de niveau de gris codée de 8 à 16 bits. Une meilleure résolution est souvent indispensable dans le domaine médical, l'astronomie et les applications d'imagerie thermique. Avec les caméras numériques, on 
"économise" une conversion analogique/numérique. La seule conversion analogique/numérique intervient dans la caméra près du capteur qui peut également être refroidi pour réduire/limiter sa sensibilité au bruit.

La plupart des systèmes de vision actuels exploitent des images en niveaux de gris. Toutefois, pour certaines applications, une image monochrome se révèle insuffisante. Une caméra et une carte d'acquisition couleurs apportent une dimension supplémentaire à l'information. Pour tirer le meilleur parti de cette information supplémentaire, il est important de comprendre ce qu'est la couleur et comment on la représente. La couleur est largement utilisée dans l'industrie automobile pour vérifier les assemblages. A cet effet, les composants des assemblages ou encore les fusibles. On utilise également la couleur pour le contrôle des cartes électroniques. Les composants, (diodes, résistances, circuits intégrés...) sont généralement insérés par un système automatisé. Le système de vision vérifie que tous les composants sont en place et bien orientés. Généralement, les données couleurs conviennent mieux à des applications pour lesquelles la couleur est l'unique caractéristique utile de l'objet. Dans les autres cas, l'information de couleur est superflue. Pour décrire, définir une couleur on utilise un modèle. Le plus répandu est le modèle RGB. Il est basé sur trois couleurs primaires (rouge, vert, bleu) qui sont combinées pour produire les autres couleurs. Dans le système RGB, les couleurs sont codées sur 24 bits soit 8 bits par couleur primaire. Par exemple, la valeur du rouge pur est 255,0,0, celle d'un gris "moyen" 128,128,128. Le système RGB est très pratique pour l'affichage des couleurs mais présente quelques inconvénients. Tout d'abord trois composantes sont nécessaires pour décrire une couleur. De plus, cette description n'est pas intuitive (naturelle). Le modèle HSL (Hue, Saturation, Luminance) est plus proche d'une description humaine. De plus, une composante (Hue ou teinte) suffit à décrire la couleur. Comme le RGB, le HSL (Hue, Saturation, Luminance) repose sur trois composantes :

- La teinte (Hue) correspond à la longueur d'onde dominante : vert, orange,... c'est à dire la couleur.

- La saturation définit la quantité de blanc ajoutée à la teinte. Le degré de saturation (de pureté) est inversement proportionnel à la quantité de blanc. Un rose par exemple est moins saturé qu'un rouge.

- La luminance représente la notion d'intensité, d'amplitude.

La teinte est très peu sensible aux variations d'éclairage, aux ombres et aux reflets. De plus, elle contient l'essentiel de l'information nécessaire pour reconnaître une couleur. Comme on n'utilise qu'une seule composante pour la reconnaissance, le traitement est plus rapide qu'en RGB mais la précision est plus faible.

Deux technologies sont utilisées dans les caméras couleur:

- Mono-CCD : des masques colorés placés devant chaque capteur CCD servent à séparer les composantes RVB.

- Tri-CCD : Pour chaque pixel de l'image, trois capteurs spécifiques à chaque composante sont sollicités. La lumière est diffractée à travers un prisme qui oriente chaque composante vers le capteur associé.

Les mono-CCD sont meilleur marché alors que les tri-CCD restituent plus précisément la couleur.

Une fois le type de caméra choisi il faut déterminer:

- Résolution du capteur? La résolution du capteur doit être choisie en fonction de la taille de l'objet et du défaut à détecter.

- Sensibilité du capteur? 


\section{Exemple 1:}

Les spécifications du cahier des charges nous imposent la détection de trous de $0.5 \mathrm{~mm}$ de diamètre sur un produit de largeur $1900 \mathrm{~mm}$. Le produit défile à une vitesse de $30 \mathrm{~m} / \mathrm{mn}$. Calculer les caractéristiques techniques du système d'acquisition

\section{Exemple 2:}

Une caméra linéaire acquiert des images de pièces posées sur un tapis roulant. La résolution dans le sens de défilement souhaitée est de $1 \mathrm{~mm}$ /pixel. On fixe donc sur un rouleau d'avancement, un encodeur qui servira à déclencher l'acquisition d'une ligne tous les $1 \mathrm{~mm}$. Si la circonférence du rouleau est de $10 \mathrm{~cm}$ combien l'encodeur devra fournir d'impulsions/sec?

\subsubsection{Autres considérations}

Les caméras et leurs capteurs sont susceptibles d'introduire un grand nombre de défauts. Diverses sources de bruit, de nature thermique et électronique, polluent le signal. Pour les atténuer, on est parfois amené à refroidir la caméra. Les éléments sensibles du capteur peuvent présenter des sensibilités différentes, ce qui a pour conséquence l'apparition d'une non-uniformité de réponse permanente. Cette dernière peut être compensée par une calibration appropriée.

Lorsque l'espacement des pixels diffère horizontalement et verticalement, on dit que l'on a affaire à des pixels non carrés. Cet effet entraine une déformation de l'image, sous forme d'un étirement. Le même étirement apparait avec les caméras linéaires, lorsque la vitesse de défilement n'est pas ajustée par rapport à la fréquence de prise d'image. De telles déformations nécessitent un ré-échantillonnage.

\subsection{Choix de la carte d'acquisition}

La carte d'acquisition dépend de la caméra choisie. Cependant il reste quelques caractéristiques à prendre en considération pour le choix de la carte d'acquisition. Ce choix réside alors dans les capacités de la carte, de la simple acquisition et transfert vers l'unité de traitement à la carte ayant des possibilités de traitements et de mémorisation avant transfert.

- Fonctions câblées.

- Composants programmables (traitements embarqués).

- Mémorisation d'images.

- Look Up Table de calibrage.

- Signaux de transfert caméra-carte.

- Entrées vidéos multiples (éventuellement simultanément dans le cas de la stéréovision).

- Déclenchement des acquisitions à partir d'un trigger externe (Entrées encodeurs).

- Sorties analogiques/numériques et tout ou rien.

- Environnement de développement.

- Bibliothèque de fonctions.

Par exemple, vaut-il mieux utiliser un système de vision sous forme d'une boîte noire externe ou bien utiliser une carte d'acquisition classique que l'on pourra synchroniser et que l'on programmera avec les outils logiciels classiques? 
Ou encore, pour certaines applications où le temps de traitement est une contrainte forte, il est nécessaire d'utiliser des cartes d'acquisition possédant des fonctions cablées.

Egalement, d'autres applications qui sont basées sur des algorithmes de comparaison nécessitent plusieurs images acquises simultanément.

Tout d'abord, on peut dimensionner la fenêtre d'acquisition communément appelée région d'intérêt (ROI). En limitant la taille de l'image acquise, on accélère le transfert vers la mémoire du PC. Il est également possible de décimer l'image directement sur la carte. La décimation est configurable par puissance de deux. Cela signifie que l'on peut décider de ne conserver sur l'image qu'un pixel sur un groupe de deux, quatre, huit ... pixels. La taille de l'image étant moins importante, on peut ainsi procéder à des transferts beaucoup plus rapides à travers le bus PCI. De plus, on peut télécharger sur la carte une table de correspondance de 256 valeurs (Look-up table) et délocaliser ainsi sur la carte une partie du traitement. Etant donné qu'à ce niveau l'image est acquise, chaque valeur pixel (niveau de gris) est traduite en une nouvelle valeur selon la "Look-Up Table". En utilisant cette technique, la carte est capable d'effectuer un seuillage matériel.

Mémoire embarquée permettant ainsi de maintenir une très haute vitesse de travail sans interruption.

Immunité par rapport au bruit: Le lien entre la caméra et la carte d'acquisition peut être source de problèmes dans un environnement bruité ou lorsqu'une grande longueur de câble est utilisée. Les signaux numériques sont plus résistants aux perturbations externes surtout lorsqu'ils utilisent un format différentiel tel que le RS-422 ou le LVDS.

Vitesse de transfert: Certaines caméras numériques peuvent avoir des capteurs de grande taille, ou des taux de rafraîchissement importants. Cela implique un débit de données très important qui peut aller au-delà de la vitesse du bus PCI (132 Mo/s en mode burst, $60 \mathrm{Mo} / \mathrm{s}$ en continu). Il est alors intéressant de pouvoir stocker les images acquises à très haute vitesse dans de la mémoire embarquée sur la carte (vitesse de transfert allant jusqu'à $200 \mathrm{Mo} / \mathrm{s}$ ) et ensuite de prendre un peu plus de temps pour rapatrier les images dans la mémoire du PC et effectuer le traitement. La mémoire embarquée est également utilisée comme FIFO "géante" lors des transferts DMA vers la mémoire du PC.

Les fonctions de "haut niveau", destinées à tous les types d'utilisateurs, permettent la détection, la configuration et l'acquisition des images. Les fonctions de "bas niveau", beaucoup plus avancées, sont elles destinées au utilisateurs ayant une meilleure maîtrise du sujet. Par exemple, les fonctions de "bas niveau" sont nécessaires si on veut valider et configurer un trigger de déclenchement et/ou d'arrêt.

La bibliothèque qui fournit une gamme très complète de fonctions pour l'analyse d'images. En plus des fonctions de la version de base, elle propose plus d'une centaine de fonctions.

\section{Développement logiciel}

Dans le développement logiciel on distingue un mode de fonctionnement hors-ligne, durant lequel un opérateur prépare la tâche d'inspection en spécifiant le type de pièces à traiter, en ajustant des paramètres de fonctionnement et en présentant éventuellement des images-échantillons de référence (ce que l'on appelle l'apprentissage).

Ensuite, le fonctionnement en-ligne, à la cadence réelle, sans intervention de l'opérateur, peut prendre place. L'acquisition des images est synchronisée avec le déplacement des pièces, et dès qu'une anomalie est détectée, une action est commandée automatiquement: éjection de la pièce en cause, arrêt de la machine, signal d'alarme, ... 


\subsection{Organisation générale des traitements}

Malgré la diversité des applications rencontrées, il est possible de dégager un schéma général pour la résolution des problèmes de vision et de définir une méthodologie appropriée. On peut décomposer les traitements en une séquence d'opérations de nature différente telle que représentées à la figure 2.

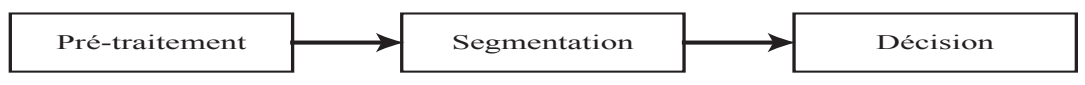

FIG. 2: Organigramme des traitements

\subsubsection{Techniques de pré-traitement}

La préparation des images peut bénéficier une vaste gamme de méthodes aptes à résoudre divers problèmes. En voici une liste largement non exhaustive :

\section{Compensation de non-uniformité d'éclairage et de réponse du capteur :}

Pour corriger cette non-uniformité des niveaux de gris il faut calibrer le système d'acquisition. Le but de ce calibrage est d'obtenir à intensité lumineuse égale une réponse identique pour chaque pixel du capteur. On obtient alors pour chaque pixel du capteur une relation linéaire en $x$, de la forme $: y=a . x+b$ avec $x$ la valeur du niveau de gris du pixel à corriger et $y$ sa valeur corrigée. Les paramètres $a$ et $b$ représentent respectivement le gain et l'offset associés au pixel. Pour déterminer le gain on présente une mire uniforme de blanc et pour l'offset on obture complétement l'objectif de manière à placer le capteur dans l'obscurité totale.

La plupart des cartes d'acquisition d'images ligne possède en standard cette correction logicielle de façon câblée sous forme de LUT (Look Up Table).

\section{Amélioration du contraste :}

Lorsque la quantité de lumière incidente est faible ou que les surfaces observées présentent des teintes voisines (en couleur comme en noir et blanc), le contraste peut être insuffisant. L'étirement d'histogramme (transformation linéaire ou non-linéaire) et son égalisation peuvent améliorer grandement la lisibilité de l'image.

\section{Amélioration de la netteté :}

Le flou peut être réduit très simplement par utilisation de filtres passe-haut, qui combinent d'une certaine façon l'image donnée et ses dérivées (rehaussement des bords), de façon à accentuer les variations au détriment des zones uniformes. Réduction de bruit par moyenne spatiale (filtres passe-bas) ou temporelle (intégration multi-images): les pixels d'une image sont normalement fortement corrélés spatialement (d'un pixel à ses voisins) et temporellement (d'un instant à l'autre). Cette corrélation peut être exploitée pour éliminer les pixels "déviants".

\section{Compensation des variations de contraste :}

En raison du vieillissement des sources d'éclairage, des conditions de lumière ambiante ou d'interventions sur les réglages du système, la quantité totale de lumière incidente peut varier. Cela peut influencer le résultat des traitements. Une façon de compenser de telles variations est la normalisation : on mesure la luminosité moyenne dans les conditions d'apprentissage, puis lors des inspections suivantes, on mesure à nouveau la luminosité moyenne de l'image et on applique un coefficient correcteur approprié. Cette technique n'est efficace que si le contenu de l'image ne change pas d'une image à l'autre. 


\section{Réalignement (correction de position et direction) :}

Pour diverses raisons, en particulier à cause du jeu mécanique de la fixation des pièces, ou un déplacement involontaire de la caméra ou des pièces elles-mêmes, ... les objets observés n'occupent pas toujours exactement la même position dans le champ de vue. Si on est capable, par des méthodes exposées ciaprès, de déterminer la position effective des pièces, on peut ramener celles-ci en position nominale par réalignement (translation et rotation, éventuellement remise à l'échelle). Cela permet de ramener l'objet inspecté dans une position telle que les régions d'intérêt utiles peuvent rester fixes.

\section{Compensation des distorsions optique, perspective, des pixels non carrés :}

Comme on l'a vu, la chaîne de formation de l'image peut introduire différents types de déformations qui donnent des objets observés une image faussée. Si on est capable de mesurer les déformations correspondantes, notamment par l'emploi de mires de formes connues grilles, matrices de points, damiers on est également capable de compenser ces déformations et de restituer la géométrie d'origine de la scène.

La plupart de techniques présentées ci-avant permettent dans une certaine mesure de réduire ou éliminer des causes d'erreurs accidentelles ou systématiques. Elles le font souvent au détriment d'autres qualités de l'image. Par exemple, la réduction de bruit s'accompagne toujours d'une perte de résolution qui se manifeste par une disparition des détails fins (à partir d'un certain niveau, et sans connaissance a priori, un algorithme ne peut pas faire la différence entre du bruit et un détail fin). Bien que le traitement numérique d'images procure une large gamme d'outils propres à réduire divers défauts, il est bien plus recommandable de les éliminer à la source, autant pour réduire la charge de calcul que pour éviter d'introduire d'autres défauts.

\subsubsection{Techniques de segmentation}

La segmentation des objets dans une image est une tâche difficile dans le cas général. On s'arrange, par des artifices d'éclairage ou autres, pour créer un contraste important entre les objets à observer et le fond. De cette façon, une simple opération de seuillage permet de les séparer. Sinon, des techniques plus élaborées doivent être employées. Ces techniques ont été traitées dans le cours de traitement d'images du tronc commun. Cependant dans le cadre d'une application de contrôle qualité les traitements utilisés doivent répondre à un certain nombre de régles.

- Les approches utilisées doivent fonctionner de façon autonome, non supervisée. Après une phase de démarrage éventuellement réalisée par un opérateur en début de production, les systèmes doivent fonctionner sans intervention pendant de longues périodes et à une cadence rapide. Ils doivent pour cela être capables de s'adapter dans une certaine mesure et résister aux changements des conditions de fonctionnement telles que l'éclairage ambiant ou la disposition des pièces.

- Les méthodes de mesure, qui peuvent être qualitatives (présent/absent, nombre, bon/mauvais...) ou quantitatives (longueur, diamètre, couleur moyenne, rugosité, ...), doivent être fiables, précises et reproductibles. Cela implique une prise en compte correcte des variations du contenu de l'image inhérentes au processus observé et une exploitation judicieuse des détails pertinents dans les images. Une solution intéressante consiste à comparer le produit en cours d'inspection à une référence. Dans ce cas les imperfections introduites par la chaîne d'acquisition peuvent être acceptées. Si les imperfections correspondent à des erreurs systématiques, c'est-à-dire se présentent toujours avec la même intensité et le même signe (distorsion optique permanente, non-uniformité stable dans le temps, désalignement constant, ...), une simple soustraction d'image, ou la comparaison à une tolérance de variation près suffit.

- Les traitements doivent suivre la cadence du temps réel imposé par la chaîne de fabrication. Étant données les hautes vitesses de défilement souhaitées (souvent comptées en pièces ou dizaines de pièces par secondes) et le gros volume de données à traiter (de l'ordre du méga-octet par image), 
des processeurs puissants combinés à des algorithmes simples et efficaces sont requis. Si nécessaire, on peut avoir recours à du matériel spécialisé pour accélérer les calculs.

- Dans la plupart des cas, on se ramène à des problèmes à deux dimensions en choisissant judicieusement le point de vue et la disposition des objets pour se ramener à des cas plans. Le travail en trois dimensions nécessite en effet une reconstruction de l'information de profondeur absente des images, qui fait appel à des techniques de triangulation spatiale telles que la stéréoscopie (emploi de deux images) ou l'éclairage structuré (motif projeté sur les objets observés). Ces techniques sont relativement délicates à mettre en oeuvre.

- On se contente également la plupart du temps d'utiliser des images en niveaux de gris. Celles-ci permettent en effet souvent de voir les caractéristiques pertinentes des objets et suffisent pour les besoins courants. D'autre part, les images en couleur sont plus volumineuses (trois fois plus en général) et les valeurs associées aux pixels doivent être vues comme des vecteurs plutôt que des scalaires. Le surcroît de complexité ainsi introduit n'est pas toujours justifiable. L'emploi de la couleur s'impose uniquement lorsqu'elle permet de révéler des contrastes invisibles en niveaux de gris. Dans la pratique, les niveaux de gris sont quantifiés sur 8 bits (256 niveaux distincts); exceptionnellement, 10 ou 12 bits sont utilisés.

- Enfin, les industriels apprécient la facilité d'utilisation avec un minimum d'intervention humaine, d'intégration dans des automatismes existants. Les méthodes et outils proposés doivent rester simples d'emploi et ne pas nécessiter les compétences de spécialistes en vision.

\subsubsection{Décision}

De cette variété d'applications, on peut néanmoins dégager un ensemble de procédés utilisés de façon récurrente et combinés pour résoudre complètement les problèmes de vision posés.

Dans une grande catégorie de cas, on ne connait pas à l'avance le contenu de l'image, ou seulement qualitativement, et on se propose de le quantifier: lorsqu'il s'agit d'objets en vrac ou de défauts imprévisibles, on est amené à les compter. Cette tâche est triviale pour des objets simples et bien contrastés. Elle peut se compliquer si les objets présentent une structure interne, qu'ils se touchent ou se chevauchent et se distinguent mal du fond et si leur aspect est très variable.

Lorsqu'on est capable d'isoler les objets de leur contexte, on peut décrire leur forme, leur taille, leur couleur, ... au moyen de mesurandes bien choisis. C'est notamment le cas de la recherche de défauts de surface, qu'ils soient contrastés, colorés ou en relief, ... On peut également faire des études statistiques de la répartition des valeurs associées. On caractérise ainsi des populations entières.

Par comparaison des paramètres mesurés avec des valeurs de référence, on peut ainsi classer les objets individuels ou populations d'échantillons en catégories pré-définies telles que "bon" et "mauvais", ou encore "bonne qualité" , "qualité moyenne" et "rejet".

Lorsqu'il s'agit de pièces de forme et dimensions bien connues à l'avance, les mesures peuvent être plus fines: un contrôle détaillé des cotes et de la forme des contours devient possible. Ces informations peuvent être comparées à des gabarits ou intervalles d'acceptation qui précisent les limites de variations tolérées.

L'utilisation de plusieurs bandes spectrales, et en particulier la traditionnelle décomposition en canaux rouge, vert et bleu, enrichit de façon notable les possibilités de mesure. En particulier, on peut se livrer à une classification beaucoup plus fine, sur la base d'une description qualitative ou quantitative de la couleur (défauts plutôt rouges, plutôt jaunes, ...). Il faut cependant rappeler que le traitement en couleur est plus exigeant à de nombreux points de vue. Il requiert en particulier que l'on se préoccupe de la stabilité des couleurs et de la fidélité de leur mesure. Par exemple, dans l'industrie de l'imprimerie, il est utile de pouvoir comparer les couleurs imprimées aux couleurs affichées à l'écran du système de 
composition de pages. On utilise pour ce faire des systèmes de représentation des couleurs indépendants du matériel.

\section{Travaux pratiques}

Les séances de manipulations de 3h chacune, sont réalisées à l'aide du logiciel Matlab. L'objectif de ces séances est de permettre aux étudiants, face à un problème concret de contrôle qualité de produit en défilement continu, de savoir définir l'architecture matériel et logicielle pour la détection de défauts.

\subsection{Scéance de $\mathbf{T p} \mathbf{N}^{o} 1$ : Calibrage et acquisition d'images ligne}

On dispose d'une bobine de film plastique qui pèse $15 \mathrm{Kg}$ et qui représente près de 200 mètres de film enroulé. La largeur du film varie suivant l'application entre 650 et $730 \mathrm{~mm}$, l'épaisseur du film est inférieure à $100 \mu \mathrm{m}$. Les propriétés des différentes couches qui composent le film complexe lui confèrent un aspect gris métal homogène. Le fournisseur n'est pas en mesure de nous garantir la qualité de son produit. Le convoyeur sur lequel vous allez travailler à les dimensions suivantes : la hauteur du portique est de $2.5 \mathrm{~m}$, la largeur est de $2 \mathrm{~m}$ et la longeur est de $2,2 \mathrm{~m}$. Un moteur triphasé permet de faire varier la vitesse de défilement du produit entre $1 \mathrm{~m} / \mathrm{mm}$ à $20 \mathrm{~m} / \mathrm{mm}$. On fournit la documentation de la carte Horizon de I2S et de la caméra linéaire. On dispose de sources d'éclairement par halogène et par tubes à néons alimentés en haute fréquence.

(1) On demande en fonction des informations sur le film plastique de choisir le type d'éclairement adéquat et la position de la caméra.

(2) La largeur de la bobine est de $670 \mathrm{~mm}$. A quelle hauteur doit être placée la caméra linéaire?

(3) On dispose de plusieurs objectifs, calculer la focale et en déduire l'objectif à utiliser. Calculer la profondeur de champ. Ne risque t-on pas une défocalisation sur les cotés?

(4) On vous demande de faire des acquisitions de lignes du produit en défilement. Pour cela, visualiser sous forme d'images en niveaux de gris et sous forme de profil le résultat de vos acquisitions. On utilise le logiciel CAP fourni avec la carte d'acquisition. Que constatez-vous sur la qualité des images lignes? Que proposez-vous comme solution, et la mettre en oeuvre. Donner alors le résultat des images ligne obtenu.

(5) On vous demande de faire des acquisitions de lignes du produit en défilement en y ajoutant vousmême des défauts. Ces acquisitions devront être sauvegardées pour être utilisées dans la séance de $\mathrm{TP} \mathrm{N}^{o} 2$.

(6) Avec le matériel à votre disposition, on vous demande de déterminer les limites du système au niveau des résolutions spatiales et temporelles.

\subsection{Scéance de $\mathbf{T p} \mathbf{N}^{o} 2$ : Détection de défauts sur produit uniforme}

Dans cette séance on vous demande de mettre en oeuvre une solution logicielle pour être en mesure de détecter et de classifier les défauts présents dans les images. On vous fournit en plus des images que vous avez acquises dans la séance TP $\mathrm{N}^{o} 1$ un base de données qui contient des images de produit uniforme en défilement (cffigure 3. On utilise pour cette séance le logiciel Matlab.

(1) Visualiser le profil de niveaux de gris de l'image en présence et en abscence de défaut. Que constatez vous? Que proposez-vous pour mettre en évidence ces variations? 
(2) On vous demande de détecter ces variations de niveau de gris correspondant aux défauts par des opérateurs differentiels à Réponse Impusionnelle Finie (RIF ou filtre non récursif) vu en cours de traitement d'images. Faire la simulation pour différentes tailles d'opérateurs

(3) On propose maintenant d'utiliser des opérateurs à Réponse Impusionnelele Infinie (RII) ou encore filtres récursifs. On fournit en annexe les équations de récurrence des opérateurs du second ordre (Deriche, Wan). Réaliser les fonctions nécessaire à la mise en oeuvre de ces opérateurs. Comparer avec les images données à la question (2) les performances de ces opérateurs.

(4) Indiquer les avantages et inconvénients de ces opérateurs RIF et RII.
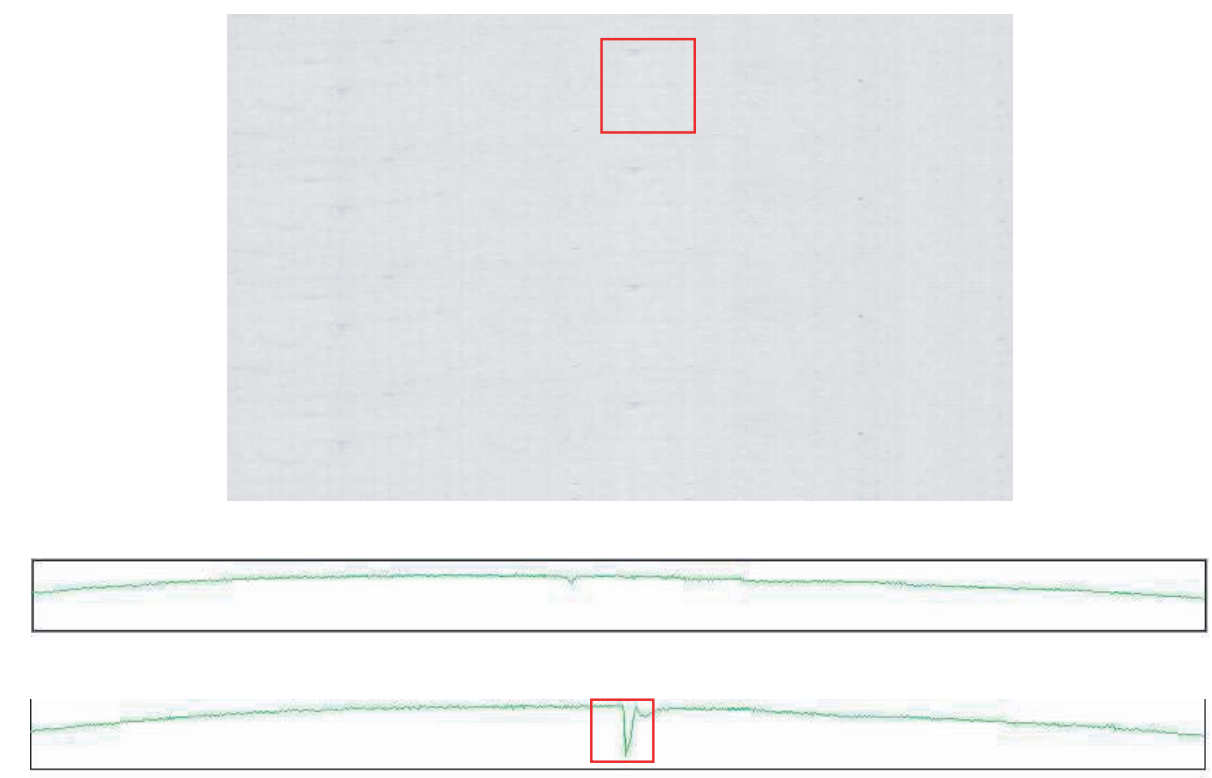

FIG. 3: Images uniformes avec défauts et images ligne correspondantes

\subsection{Scéance de $\mathbf{T p} \mathbf{N}^{o} 3$ : Détection de défauts sur produit texturé}

Dans cette séance on vous demande de mettre en oeuvre une solution logicielle pour être en mesure de détecter et de classifier les défauts présents dans les images. On vous fournit une base de données qui contient des images de produit texturé (cffigure 4). On utilise pour cette séance le logiciel Matlab.

(1) Dans une $1^{r e}$ partie on vous donne les grandeurs statistique du $1^{\text {er }}$ et du $2^{\text {nd }}$ ordre sur une banque d'images sans défauts pour différents voisinage: 20pixels*12lignes, 40pixels*25lignes, 80pixels*50lignes. Calculer pour vos images au voisinage du défaut les grandeurs statistiques du $1^{e r}$ et du $2^{\text {nd }}$ ordre. Que constatez-vous?

(2) Modifier la taille du voisinage et reprendre la question précédente. Que peut on dire sur la taille de ce voisinage?

(3) Quelle est la relation entre la taille du voisinage et la taille du défaut?

(4) Réaliser les fonctions permettant de détecter les défauts. Si maintenant on a une variation de niveaux de gris (par exemple éclairage non constant), que se passe t-il? Que faut il prendre en compte?

(5) Dans cette partie on ne connaît pas les caractéristiques statistique du $1^{e r}$ et du $2^{\text {nd }}$ ordre de l'image de référence. Proposer une démarche qui permette de détecter les défauts présents dans l'image. 

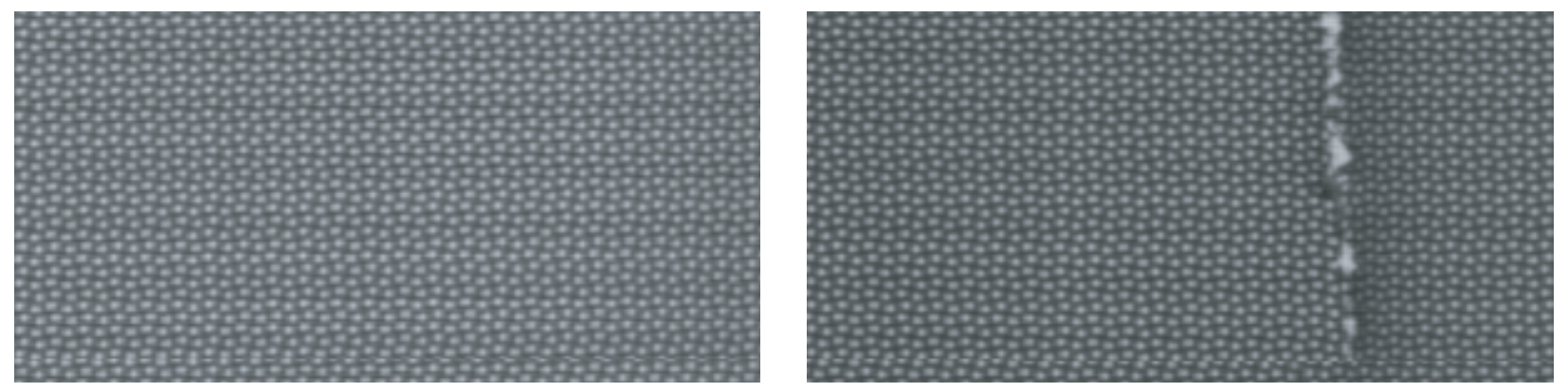

FIG. 4: Images texturées sans et avec défauts

\section{Références}

[1] A.Taleb-Ahmed, Cours, TD et TP de traitement d'images de maîtrise EEA, Productique et de Physique à Université du Littoral Cote d'Opale à Calais.

[2] BASSEVILLE and All, Fiches d'algorithmes de segmentation des signaux. Traitement du signal, 9, pages 115-147, 1992.

[3] R.M. HARALICK. Image segmentation techniques. Computer Vision Graphics and Image Processing, pages 100-132, 1985.

[4] J.P. COCQUEREZ et S. PHILIPP. Analyse d'images : filtrage et segmentation. Masson, 1995.

[5]S.U. LEE et S.Y. CHUNG. A comparative performance study of several global thresholding techniques for segmentation. CVGIP, 52, pages 171-190, 1990.

[6] P.K. SAHOO et S. SOLTANIS. A survey of thresholding techniques. CVGIP, 4, pages 233-260, 1988.

[7] M.T. HUECKEL. An operator which locates edges in digitized pictures, J. Ass. Comput. Mach., Vol. 18, p. 113-125, 1971.

[8] L.G. ROBERTS. Machine perception of three-dimensional solids. Optical and Electro-optical Information Processing, pages 159-197, 1965.

[9] J.M.S. PREWITT. Objet enhancement and extraction. Pictures Processing and Psychopictorics, 1970.

[10] R. KIRSCH. Computer determination of the constituent structure of biological images, Computers and Biomedical Research, Vol. , No 3, pages 315-328, 1978.

[11] J. CANNY. A computational approach to edge detection. IEEE Transaction on Pattern Analysis and Machine Intelligence, 8, pages 679-698, 1986.

[12] R. DERICHE. Fast algorithms for low-level vision. IEEE Transaction on Pattern Analysis and Machine Intelligence, 12, pages 78-87, 1990.

[13] R. DERICHE. Recursively implementing the gaussian and its derivatives. Technical Report nř 1893, INRIA, Unité de Recherche Sophia-Antipolis, 1993.

[14] R. DERICHE, O. FAUGERAS, R. VAILLANT. Four applications of differential geometry to computer vision. In Guy Orban and H-H. Nagel, editors, Artificial and Biological Vision Systems, Basic Research Series, pages 93-141. Springer-Verlag, 1993.

[15] Y.F. WAN. Un opérateur hyperbolique pour la détection de contours dans les images numériques. Thèse de 3ème cycle, Université des Sciences et Technologies de Lille, 1996.

[16] J. CARON. Les systèmes de vision linéaire pour le contrôle qualité dans les procédés de production 
continue. Thèse de troisième cycle, Université du Littoral, décembre 2000.

[17] V. STOCLIN. Evaluation des filtres de détection de contours, du second ordre et sous forme récursive, à l'aide de critères discrets. Thèse de Doctorat en Automatique et Informatique Industrielle, UST de Lille 1, 2000.

[18] E.I. JURY. Theory and application of the Z-transform method. Wiley, N.Y, 1964.

[19] J. SERRA. Image analysis and mathematical morphology. Academic press, 1982.

[20] M. COSTER et J.L. CHERMANT. Précis d'analyse d'images. CNRS, 1989.

[21] J.S.J. LEE, R. HARALICK et L.G. SHAPIRO. Morphological edge detection. IEEE Journal on Robotics and Automation, 3(2), pages 142-155, 1987.

[22] A. ROSENFELD et E. TROY. Visual texture analysis. Tecnhical Report TR-116, Computer science center, University of Maryland, 1970.

[23] M. UNSER et F.DE COULON. Detection of defects by texture monitoring in automatic visual inspection. In 2nd Int. Conf. On Robot Vision and Sensory Controls, Stuttgart, Germany, 1982.

[24] R.M. HARALICK. Texture features for image classification. IEEE Trans. Syst. Man. Cybern., 3 , pages $610-621,1973$.

[25] R.M. HARALICK. Statistical and structural approaches to texture. IEEE Trans. Syst. Man. Cybern., 67, pages 786-804, 1979.

[26] M. UNSER. Description statistique de texture: Application à l'inspection automatique. Thèse de 3ème cycle, EPFL, Lausanne, 1984.

[27] Y. CHEN. Analyse d'images pour la détection de verglas. Thèse de 3ème cycle, Université de Paris-Sud, Centre d'Orsay, 14 Mars 1991.

[28] L. Duvieubourg, A .Taleb-Ahmed, J.G. Postaire, Analyse of recursive filters for the detection of defects on flat product, QCAV'99, pp, juil 1999, Trois riviére, Canada. 